\title{
Association between Elder Mistreatment and Suicidal Ideation among Community-Dwelling Chinese Older Adults in the USA
}

\author{
XinQi Dong ${ }^{a}$ Ruijia Chen ${ }^{a}$ Bei Wu $^{b}$ Ning Jackie Zhang ${ }^{c}$ \\ Ada Chan Yuk-Sim Muid ${ }^{d}$ Iris $\mathrm{Chi}^{\mathrm{e}}$

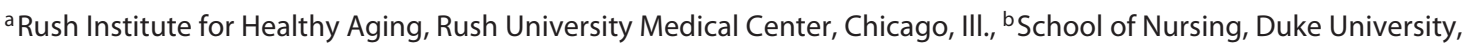 \\ Durham, N.C., 'College of Health and Public Affairs, University of Central Florida, Orlando, Fla., ${ }^{\mathrm{d}}$ School of \\ Social Work, Columbia University, New York, N.Y., and eSchool of Social Work, University of Southern California, \\ Los Angeles, Calif., USA
}

\section{Key Words}

Suicidal ideation - Elder mistreatment . Chinese .

Older adults

\begin{abstract}
Background: Elder mistreatment and suicidal ideation are important public health concerns among aging populations. However, very few studies have been conducted to explore the association between elder mistreatment and suicidal ideation. Objectives: To examine the association between elder mistreatment and suicidal ideation among Chinese older adults in the USA. Methods: Guided by a community-based participatory research approach, in this study we conducted in-person interviews with Chinese older adults aged 60 years and older in the Greater Chicago area from 2011 to 2013. Elder mistreatment was assessed by a 10-item instrument derived from the Hwalek-Sengstock Elder Abuse Screening Test (H-S/EAST) and the Vulnerability to Abuse Screening Scale (VASS). Suicidal ideation was assessed by the ninth item of the Patient Health Questionnaire-9 (PHQ-9) and the Geriatric Mental State Examination-Version A (GMS-A). Results: Overall, 3,159 Chinese older adults participated in this study, and their mean age was 72.8 years. After controlling for age, gen-
\end{abstract}

der, education, income, medical comorbidities, depressive symptoms, and social support, elder mistreatment was significantly associated with 2-week suicidal ideation (OR 2.46, $95 \% \mathrm{Cl} 1.52-4.01$ ) and 12-month suicidal ideation (OR 2.46, $95 \% \mathrm{Cl} 1.62-3.73)$. With respect to gender differences, the study found that the association remained significant for older women but not for older men after adjusting for all confounding factors. Conclusion: As the largest epidemiological study conducted among Chinese older adults in the USA, this study suggests that elder mistreatment is significantly associated with 2-week and 12-month suicidal ideation in older women but not in older men. Longitudinal studies should be conducted to explore the mechanisms through which elder mistreatment links with suicidal ideation.

(c) 2015 S. Karger AG, Basel

\section{Introduction}

Elder mistreatment, which refers to the intentional actions that result in harm or create a serious risk of harm to a vulnerable older adult or a caregiver's failure to satisfy the elder's basic needs and safe living conditions [1], is a growing public health and human rights issue. It is

\section{KARGER}

E-Mail karger@karger.com

www.karger.com/ger
(C) 2015 S. Karger AG, Basel

0304-324X/15/0621-0071\$39.50/0
Dr. XinQi Dong

Rush Institute for Healthy Aging, Rush University Medical Center

1645 West Jackson, Suite 675

Chicago, IL 60612 (USA)

E-Mail xinqi_dong@rush.edu 
suggested that 1 in 10 older adults has suffered from elder mistreatment in the previous year [2]. The existing literature, albeit limited, has shown that elder mistreatment may lead to physical and cognitive functional declines [3, $4]$, mental health problems [5, 6], and mortality [7]. Although previous research has documented potential adverse outcomes of elder mistreatment, there is a dearth of research on its association with suicidal ideation, a growing health issue among older adults.

Due to age-related declines in physical function and mental health as well as decreases in social networks, older adults are prone to a high risk of suicidal ideation [810]. Research has indicated that the worldwide prevalence of suicidal ideation in the previous month in older adults ranges from 2.3 to $15.9 \%$, depending on the methodologies used such as study settings, measurements, and recruitment strategies $[11,12]$. Suicidal ideation has been found to be a significant risk factor for both suicide attempts and completed suicide among older adults. For example, Waern et al. [13] studied 85 elderly individuals aged 65 years and older who died by suicide and found that $28.2 \%$ of them had had suicidal ideation during the year proceeding suicide. In order to prevent suicidal behavior among older adults, knowledge of factors associated with suicidal ideation is crucial.

The extant literature has documented a variety of sociodemographic, physical, and mental health factors that are associated with suicidal ideation $[8,10,14]$. Using data from the Canadian Community Health Survey, Corna et al. [8] found that being male, being younger, being widowed, having lower social support, and having higher psychological distress were associated with an increased likelihood of suicidal ideation among adults aged 55 years and older. A cross-sectional study of Chinese older adults aged 60 years and above in Hong Kong suggested that lower physical function, poor mental health, and financial and relationship problems were associated with an increased risk for suicidal ideation [10]. In a study of 1,061 older patients aged 60 years and over in Australia, depressive feelings were significantly associated with suicidal ideation [9]. A growing body of literature has also examined the association between traumatic experience and suicidal ideation. This line of research consistently suggests that traumatic events have a positive association with victims' suicidal ideation [15-17]. Further, a crossnational analysis of data from the WHO World Mental Health Survey demonstrates that among traumatic events, interpersonal violence had the strongest effect on suicidal behavior [18]. Unfortunately, these studies primarily focused on child abuse and intimate partner violence
[19], whereas the association between elder mistreatment and suicidal ideation has not yet been well established.

The interplay of elder mistreatment and suicidal ideation may vary by cultural, racial, and ethnic backgrounds. In Confucian philosophy, where harmony is highly valued, unfavorable interpersonal relationships may carry a stigma and decrease older adults' self-esteem to a large extent. Earlier studies that examined the association between interpersonal relationships and suicidal ideation have been focused on family dysfunction [10, 20]. Little research, however, has been conducted to understand the association between elder mistreatment and suicidal ideation. To the best of our knowledge, only one study explored the association between elder mistreatment and suicidal ideation in Chinese older adults. Wu et al. [21] conducted a survey with 2,039 Chinese older adults and found that elder mistreatment was associated with an increased risk for suicidal ideation. Nevertheless, the study was restricted to older adults in rural China, and thus the finding may not be generalizable to Chinese older adults in the USA, who have different socioeconomic backgrounds, are enrolled in different health care systems, and tend to be confronted with significant linguistic and cultural barriers [22-26].

Although there is evidence that suicidal ideation and elder mistreatment are prevalent among Chinese older adults in the USA $[27,28]$, we are unaware of any study that examines the association between elder mistreatment and suicidal ideation in this population. Further, prior studies documented that Chinese older women were more likely to experience suicidal ideation than older men $[10,29]$, implying that there may be gender differences in factors associated with suicidal ideation. Building upon this previous study, the purposes of this study were to (1) explore the association between elder mistreatment and suicidal ideation among Chinese older adults in the USA and (2) understand gender differences with respect to the association between suicidal ideation and elder mistreatment.

\section{Subjects and Methods}

\section{Population and Settings}

The Population Study of Chinese Elderly in Chicago (PINE) is a community-engaged, population-based epidemiological study of US Chinese older adults aged 60 years and over in the Greater Chicago area. The project was initiated by a synergistic communityacademic collaboration between the Rush Institute for Healthy Aging, Northwestern University, and many community-based social service agencies and organizations throughout the Greater Chicago area.
Dong/Chen/Wu/Zhang/Mui/Chi 
Study Design and Procedure

In order to ensure the study's relevance to the well-being of the Chinese community and increase community participation, the PINE study implemented extensive culturally and linguistically appropriate community recruitment strategies strictly guided by a community-based participatory research approach. The formation of this community-academic partnership allowed us to develop an appropriate research methodology in accordance with the local Chinese cultural context, in which a community advisory board plays a pivotal role in providing insights and strategies for conducting research. Board members were community stakeholders and residents. Over 20 social service agencies, community centers, health advocacy agencies, faith-based organizations, senior apartments, and social clubs served as the basis for study recruitment sites.

Community-dwelling older adults aged 60 years and over who self-identified as Chinese were eligible participants in the study. Out of 3,542 eligible older adults approached, 3,159 agreed to participate in the study from 2011 to 2013, yielding a response rate of $91.9 \%$. In order to ensure cultural and linguistic sensitivity, trained multicultural and multilingual interviewers conducted face-toface home interviews with the participants in their preferred language and dialects, such as English, Cantonese, Taishanese, Mandarin, or Teochew. Based on the available census data drawn from the 2010 USA Census and a random block census project conducted in the Chinese community in Chicago, the PINE study is representative of the Chinese aging population in the Greater Chicago area [30]. The study was approved by the Institutional Review Boards of the Rush University Medical Center. Further in-depth details of the PINE study's design are published elsewhere [31].

\section{Measurements}

Independent Variable: Elder Mistreatment

The elder mistreatment instrument used in this study was derived from items first developed by Hwalek and Sengstock [the Hwalek-Sengstock Elder Abuse Screening Test (H-S/EAST)] in 1986 [32] and then modified by Schofield and Mishra [the Vulnerability to Abuse Screening Scale (VASS)] in 2003 [33]. The modified VASS has been administered to Chinese elderly populations both in mainland China and the USA $[34,35]$. Our participants were asked if they had had the following experience after they turned 60 years old: (1) family conflicts at home; (2) felt uncomfortable with someone in the family; (3) felt that nobody wanted them around; (4) been told by someone that they caused too much trouble; (5) been afraid of someone in the family; (6) felt that someone close tried to hurt or harm them; (7) been neglected or confined; (8) been called a name or put down; (9) been forced by someone to do things, or (10) someone took belongings without permission. A 'yes' response to any of the above questions was considered as having experienced elder mistreatment in the present study. The scale demonstrated good reliability in this study sample, with Cronbach's a of 0.80 .

\section{Dependent Variable: Suicidal Ideation}

Given that prior research has shown that factors associated with suicidal ideation during extended periods of time may be different from more recent ones [36], this study assessed suicidal ideation in the previous 2 weeks and in the last 12 months to better elucidate the association between elder mistreatment and suicidal ideation. Suicidal ideation in the previous 2 weeks was assessed by the ninth item of the Patient Health Questionnaire-9 (PHQ-9), a screening instrument for depressive symptoms over the past 2 weeks [37]. Participants were asked how often they thought they would be better off dead or about hurting themselves in some way over the last 2 weeks. Questions were categorized as: (1) not at all; (2) several days; (3) more than half of the days, and (4) nearly every day. Any affirmative response to options $2-4$ was defined as having 2 -week suicidal ideation. Suicidal ideation in the past 12 months was measured by the Geriatric Mental State Examination-Version A (GMS-A), which is a semi-structured interview guide designed for the elderly [38]. Participants were asked: 'Have you ever felt suicidal or wished to be dead in the past 12 months?' The question elicited a 'yes' or 'no' answer. A 'yes' response to the above question classified a respondent as having 12-month suicidal ideation. The Chinese version has been validated in earlier studies [10].

\section{Confounding Variables}

Sociodemographic Characteristics. Basic sociodemographic information included age (years), gender, years of education, and annual personal income.

Medical Comorbidities. To assess medical comorbidities, the participants were asked if they had ever been told by a doctor, nurse, or therapist that they had: (1) heart disease, heart attack, coronary thrombosis, coronary occlusion, or myocardial infarction; (2) stroke or brain hemorrhage; (3) cancer, malignancy, or a tumor of any type; (4) high cholesterol; (5) diabetes, sugar in the urine, or high blood sugar; (6) high blood pressure; (7) a broken or fractured hip; (8) thyroid disease, or (9) osteoarthritis, inflammation, or problems with joints. The number of medical comorbidities was calculated by totaling the number of 'yes' responses to the nine items listed earlier.

Depressive Symptoms. We used the PHQ-9 to assess depressive symptoms among Chinese older adults. The PHQ-9 consists of nine items; each of them assesses the nine Diagnostic and Statistical Manual of Mental Disorders (DSM-IV) depression symptom criteria [37]. Participants were asked if they had the following symptoms in the last 2 weeks: (1) changes in sleep; (2) changes in appetite; (3) fatigue; (4) feelings of sadness or irritability; (5) loss of interest in activities; (6) inability to experience pleasure or feelings of guilt or worthlessness; (7) inability to concentrate or make decisions, and (8) feeling restless or slowed down. The ninth item of the PHQ-9 was used to assess 2 -week suicidal ideation, as described previously. Respondents indicated answers to each question on a 4-point scale ranging from $0=$ 'not at all' to $3=$ 'nearly every day'. Participants who indicated that their answers were other than 'not at all' to any of the eight questions were considered as having depressive symptoms. The PHQ-9 has been validated among Chinese Americans and has good interrater reliability [39]. The standardized Cronbach $\alpha$ of the PHQ-9 in the PINE study was 0.82 .

Social Support. We assessed the current levels of social support by asking about both the positive and the negative aspects of social support. Regarding its positive aspects, the participants were asked how frequently they were able to open to or seek help from their spouses, family members, and friends. As for negative social support, the participants were asked how frequently they felt too much was demanded from them or that they were criticized by their spouses, family members, and friends. Respondents indicated answers to each question on a 3 -point scale ranging from $1=$ 'hardly ever' to 3 = 'often'. Negative aspects of social support were recoded as $1=$ 'often' to 3 = 'hardly ever'. Social support was calculated as the sum of all twelve items. 
Table 1. Comparison of the characteristics of the study participants by suicidal ideation

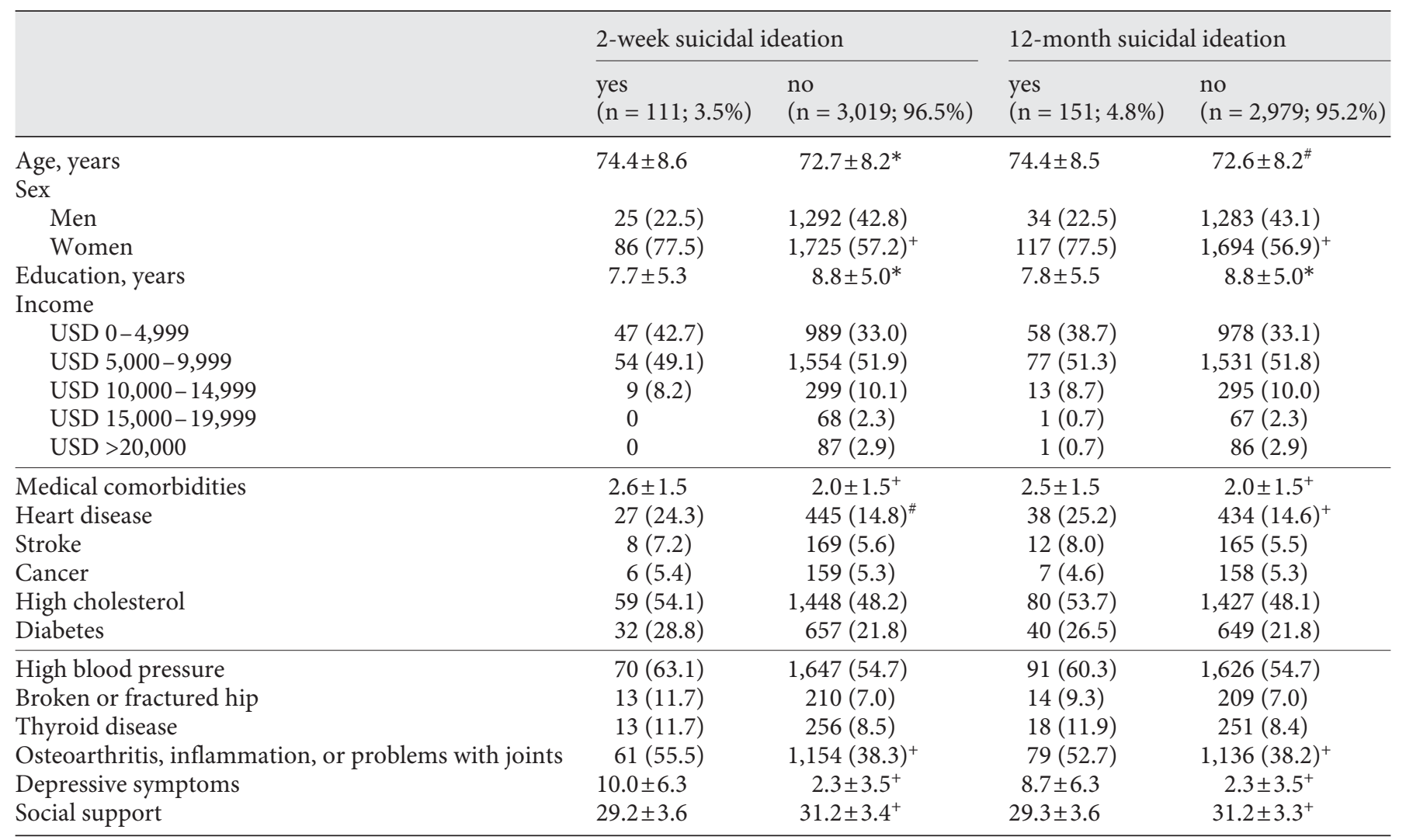

Values are presented as means \pm SD or $\mathrm{n}(\%) .{ }^{*} \mathrm{p}<0.05,{ }^{*} \mathrm{p}<0.01,{ }^{+} \mathrm{p}<0.001$.

Data Analysis

We first used $\chi^{2}$ and $t$ tests to compare sociodemographic characteristics, medical comorbidities, depressive symptoms, social support, and elder mistreatment between older adults with and those without 2 -week and 12-month suicidal ideation among the general populations. We then separated the analysis by gender to understand the sociodemographic and socioeconomic characteristics in women and in men, respectively. To examine the association between elder mistreatment and suicidal ideation, we utilized logistic regression models to control for potential confounding factors, which were drawn from the literature review $[10,14,21]$. Model A was adjusted for basic sociodemographic characteristics, including age and sex. The next model (model B) included additional socioeconomic variables, such as education and income. In model $\mathrm{C}$, we added the number of medical comorbidities to the previous model. We then added depressive symptoms as a potential confounder to model D. In the final model (model E), we added social support and all other confounders to the analysis, with suicidal ideation as the dependent variable.

ORs, 95\% CIs, and $\mathrm{p}$ values are reported for the multivariate analyses. These analyses was then repeated for men and women separately. The Hosmer-Lemeshow goodness-of-fit test was used to assess the overall goodness of fit of the models. All statistical analyses were conducted using SAS version 9.2 (SAS Institute Inc., Cary, N.C., USA).

\section{Results}

\section{Characteristics of All Participants by the Presence of} Suicidal Ideation

Overall, 1,862 older women (58.9\%) and 1,297 older men $(41.1 \%)$ participated in this study, and the average age of all participants was 72.8 years. A total of $4.7 \%$ of the older women and $1.9 \%$ of the older men reported 2 -week suicidal ideation; $6.5 \%$ of the older women and $2.6 \%$ of the older men had 12-month suicidal ideation. The prevalence of elder mistreatment among older women and older men was 15.8 and $14.3 \%$, respectively.

Older adults with 2 -week suicidal ideation were more likely to be older, be women, have lower educational levels, have more medical comorbidities, have heart disease, report osteoarthritis, inflammation, or problems with joints, have higher levels of depressive symptoms, and have lower social support.

Likewise, older adults who experienced 12-month suicidal ideation were more likely to be older, be women, have lower educational levels, have more medical comor- 
Table 2. Comparison of the characteristics of the study participants by suicidal ideation in older women and men

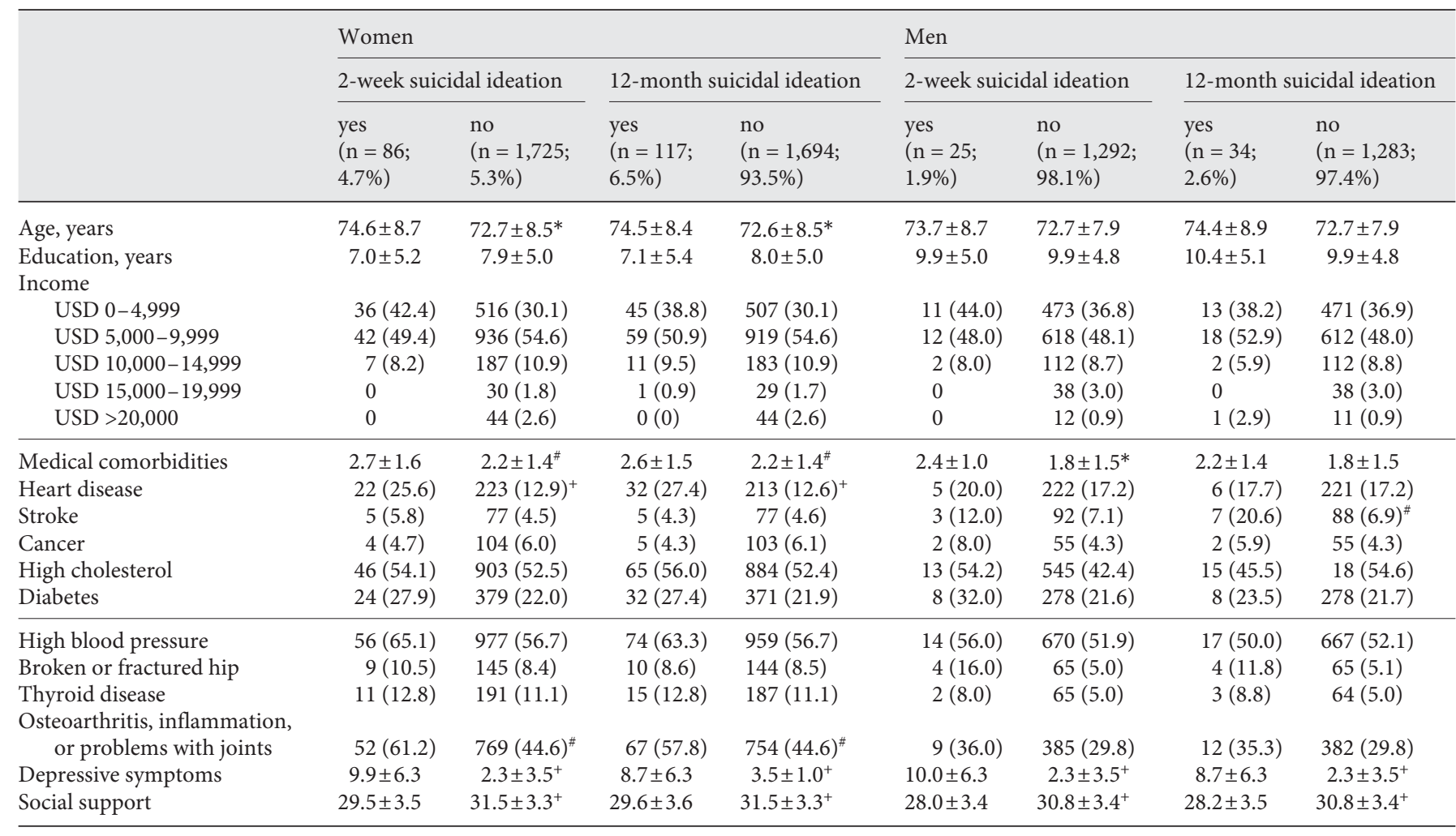

Values are presented as means $\pm \mathrm{SD}$ or $\mathrm{n}(\%) .{ }^{*} \mathrm{p}<0.05,{ }^{*} \mathrm{p}<0.01,{ }^{+} \mathrm{p}<0.001$.

bidities, have heart disease, report osteoarthritis, inflammation, or problems with joints, and have higher levels of depressive symptoms and lower social support (table 1).

Characteristics of Older Women and Men by the

Presence of Suicidal Ideation

Chinese older women with 2 -week suicidal ideation differed significantly from those without suicidal ideation by age, number of medical comorbidities, heart disease, osteoarthritis, inflammation, or problems with joints, depressive symptoms, and social support. On the other hand, older women with 12-month suicidal ideation were more likely to be of older age, have heart disease, have more medical comorbidities, have osteoarthritis, inflammation, or problems with joints, report greater levels of depressive symptoms, and have lower social support.

Older men with 2 -week suicidal ideation were more likely to have a greater number of medical comorbidities, endorse depressive symptoms, and have lower social support than those without 2-week suicidal ideation. Compared with older men without 12 -month suicidal ide- ation, those with 12-month suicidal ideation were more likely to have strokes, depressive symptoms, and lower social support (table 2 ).

\section{Prevalence of Elder Mistreatment by the Presence of Suicidal Ideation}

The prevalence of elder mistreatment by suicidal ideation is shown in table 3 . Older adults who experienced 2-week ( 42.7 vs. $14.1 \%, \mathrm{p}<0.001$ ) and 12 -month suicidal ideation (40.7 vs. $13.9 \%, \mathrm{p}<0.001)$ had a significantly higher prevalence of elder mistreatment than those without suicidal ideation.

In older women, the prevalence of elder mistreatment among those with 2 -week ( 42.4 vs. $14.5 \%, \mathrm{p}<0.001)$ and 12 -month suicidal ideation ( 40.5 vs. $14.1 \%, \mathrm{p}<0.001$ ) was significantly higher than among those without suicidal ideation. Similar findings were found in older men: those who experienced 2 -week ( 44.0 vs. $13.7 \%, \mathrm{p}<0.001$ ) and 12 -month suicidal ideation (41.2 vs. $13.6 \%$, p < 0.001 ) also reported significantly higher rates of elder mistreatment. 
Table 3. Elder mistreatment by the presence of suicidal ideation in older adults

\begin{tabular}{|c|c|c|c|c|c|c|}
\hline & \multicolumn{3}{|c|}{ 2-week suicidal ideation } & \multicolumn{3}{|c|}{ 12-month suicidal ideation } \\
\hline & yes & no & $\mathrm{p}$ & yes & no & $\mathrm{p}$ \\
\hline \multicolumn{7}{|c|}{ Total participants } \\
\hline \multicolumn{7}{|c|}{ Elder mistreatment } \\
\hline Yes & $47(42.7)$ & $425(14.1)$ & & $61(40.7)$ & $411(13.9)$ & \\
\hline No & $63(57.3)$ & $2,581(85.9)$ & $<0.001$ & $89(59.3)$ & $2,555(86.1)$ & $<0.001$ \\
\hline \multicolumn{7}{|l|}{ Women } \\
\hline \multicolumn{7}{|c|}{ Elder mistreatment } \\
\hline Yes & $36(42.4)$ & $249(14.5)$ & & $47(40.5)$ & $238(14.1)$ & \\
\hline No & $49(57.7)$ & $1,471(85.5)$ & $<0.001$ & $69(59.5)$ & $1,451(85.9)$ & $<0.001$ \\
\hline \multicolumn{7}{|l|}{ Men } \\
\hline \multicolumn{7}{|c|}{ Elder mistreatment } \\
\hline Yes & $11(44.0)$ & $176(13.7)$ & & $14(41.2)$ & $173(13.6)$ & \\
\hline No & $14(56.0)$ & $1,109(86.3)$ & $<0.001$ & $20(58.8)$ & $1,103(86.4)$ & $<0.001$ \\
\hline
\end{tabular}

Table 4. Association between overall elder mistreatment and suicidal ideation

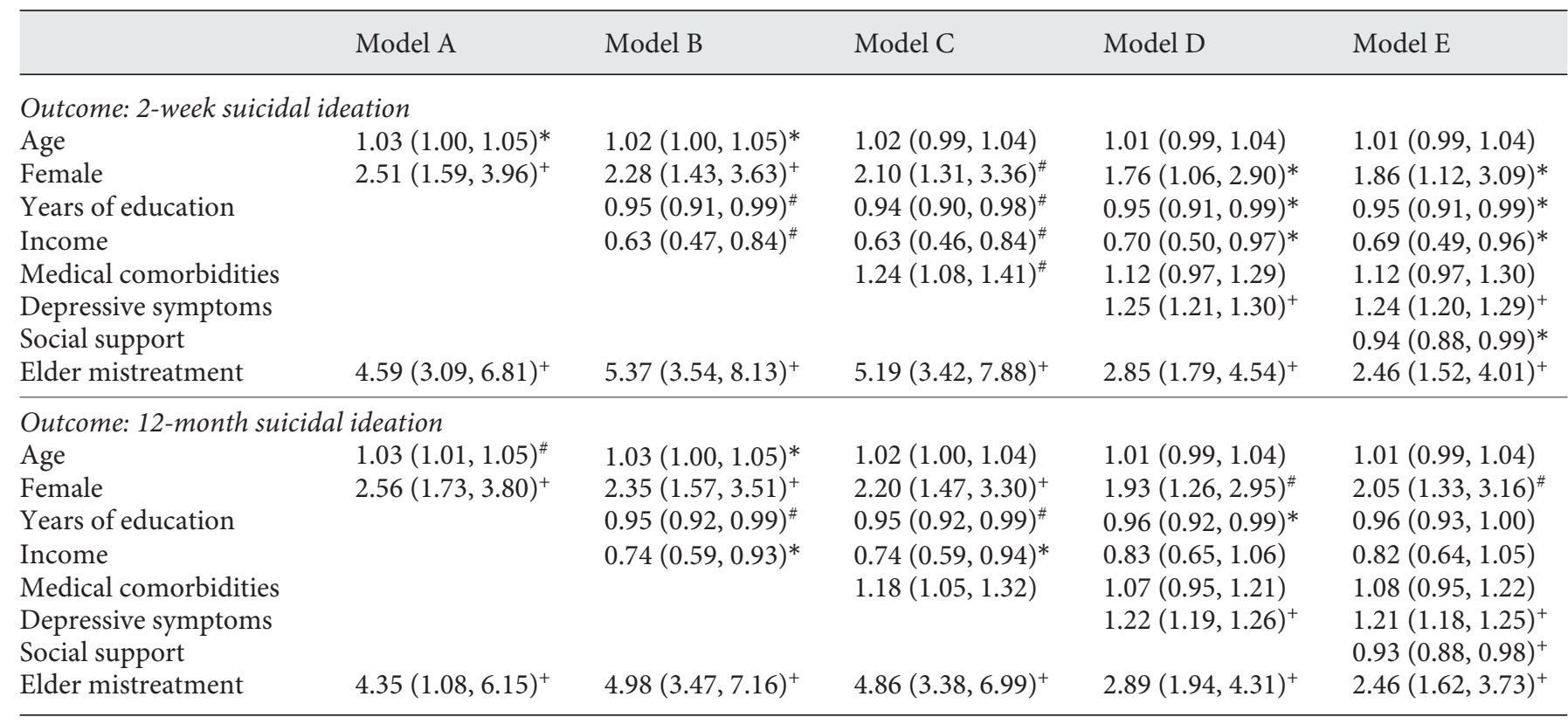

Values denote OR (95\% CI). ${ }^{*} \mathrm{p}<0.05,{ }^{*} \mathrm{p}<0.01,{ }^{+} \mathrm{p}<0.001$.

\section{Association between Elder Mistreatment and}

2-Week and 12-Month Suicidal Ideation among

All Participants

The association between elder mistreatment and 2-week and 12-month suicidal ideation among all par- ticipants is presented in table 4 . In the full model that controlled for age, gender, years of education, income, medical comorbidities, depressive symptoms, and social support, older adults who experienced elder mistreatment were 2.46 times more likely to report 2 -week sui- 
Table 5. Association between elder mistreatment and suicidal ideation in older women and men

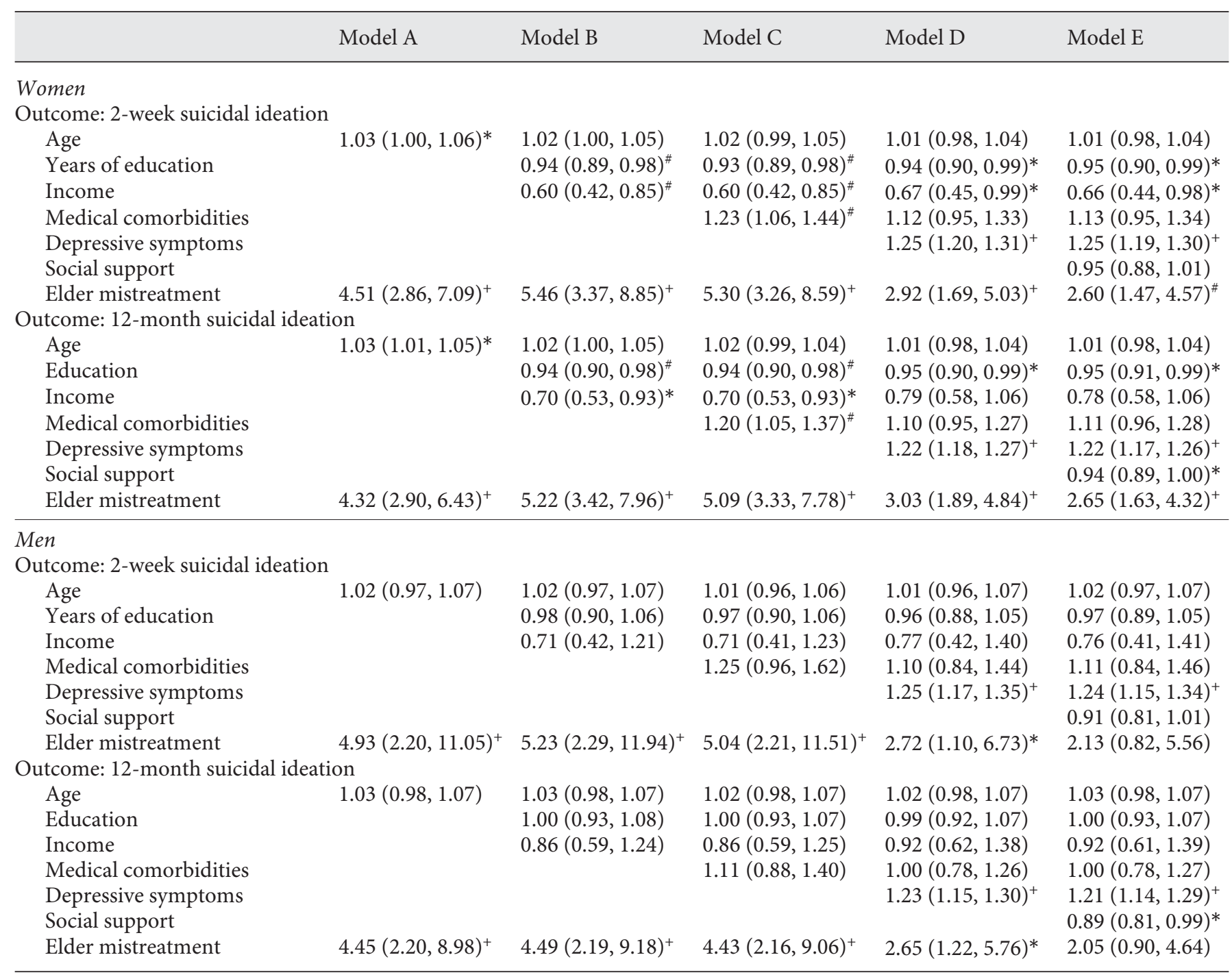

${ }^{*} \mathrm{p}<0.05,{ }^{\#} \mathrm{p}<0.01,{ }^{+} \mathrm{p}<0.001$.

cidal ideation (OR 2.46, 95\% CI 1.52-4.01) and 2.46 times more likely to experience 12 -month suicidal ideation (OR 2.46, 95\% CI 1.62-3.73) than those who did not experience elder mistreatment. The model fit indexes of the data were good (Hosmer-Lemeshow test: $\mathrm{p}>$ 0.05).

\section{Association between Elder Mistreatment and Suicidal Ideation in Older Women and Men}

Table 5 presents the association between elder mistreatment and suicidal ideation in older women and men. After adjusting for age, gender, education, income, med- ical comorbidities, depressive symptoms, and social support, older women suffering elder mistreatment were more likely to experience 2-week (OR 2.60, 95\% CI 1.474.57 ) and 12-month suicidal ideation (OR 2.65, 95\% CI 1.63-4.32) than older women not suffering elder mistreatment.

However, among older men, after adding social support to the final model, no significant association between elder mistreatment and suicidal ideation was found, indicating that social support may mediate this association in older men. 


\section{Discussion}

This study, to our knowledge, is the first to examine the association between elder mistreatment and suicidal ideation among Chinese older adults in the USA. In this study, elder mistreatment was significantly associated with increased risk for 2-week and 12-month suicidal ideation among all the participants. However, of greater interest, after adding social support to the full model, the association remained significant for older women but not for older men. The findings of this study provide important empirical support for possible preventive measures and interventions for reducing suicidal ideation among US Chinese older populations.

Elder mistreatment appears to be significantly associated with suicidal ideation after controlling for several important confounders. This finding is consistent with that of a study conducted in Hubei, China, which suggested that elder mistreatment was a risk factor for suicidal ideation among 2,039 Chinese older adults [21]. Several potential mechanisms may help explain the association between elder mistreatment and suicidal ideation among Chinese older adults. In a culture where respect for elders is highly valued, elder mistreatment may evoke feelings of shame, decrease older adults' self-esteem, and raise thoughts that life is not worth living. In addition, older adults who experience elder mistreatment may be less likely to engage in social activities, which may increase their risk of loneliness and depression $[5,40]$ and ultimately lead to suicidal ideation. This may be especially true for immigrant older adults. Since many immigrant older adults, especially newcomers, may have experienced a decline in size of their social network during the course of immigration, they may depend more on their family members or trusted friends. Thus, if victimized by their trusted ones, these older adults may feel more helpless and are less able to cope with the stress. Another possible explanation is that abusive acts may induce physical injuries that could potentially exacerbate physical functional declines and decrease older adults' willingness to live [3]. The findings also demonstrate that the degree of the association between elder mistreatment and suicidal ideation seems to be influenced greatly by depressive symptoms. Further studies may need to explore how depressive symptoms interact with elder mistreatment and suicidal ideation among this population.

Interestingly, elder mistreatment was associated with 2-week and 12-month suicidal ideation in older women but not in older men. This finding is in contrast to a study conducted in Sweden, in which elder mistreatment was associated with a greater risk for suicidal ideation in old- er men than in older women [41]. In Chinese culture, women tend to play subordinate roles, which may expose them to financial, educational, and employment disadvantages. Therefore, when being mistreated, older women may have fewer resources for recovery than older men. Compared with Chinese older men, Chinese older women may also have less effective coping strategies. For example, when facing life stress and trauma, women tend to employ passive coping strategies such as rumination, while men are more likely to use active coping strategies [42]. Therefore, elder mistreatment may have a more deleterious impact on women's mental health. It should be noted that elder mistreatment was associated with suicidal ideation in older men when controlling for sociodemographic characteristics, medical comorbidities, and depressive symptoms. However, after adding social support, no significant association was found, indicating that social support may protect male elder mistreatment victims from suffering from suicidal ideation.

The findings of this study should be interpreted in light of several limitations. First, elder mistreatment and suicidal ideation were both self-reported, which may be subject to reporting bias. Of importance is the fact that our findings are strengthened by the utilization of the community-based participatory research approach, which enabled us to gain the trust of the participants and thus might have helped us reduce any potential reporting bias. Second, this study used a cross-sectional design, and we were unable to clarify the time sequence of elder mistreatment and suicidal ideation and to establish the temporal association between elder mistreatment and suicidal ideation. Future longitudinal studies should elucidate the mechanisms through which elder mistreatment links with suicidal ideation. Third, although this study adjusted for potential confounders, there might be unknown or unmeasured confounders that we failed to include in the analysis. Finally, we did not examine the association between elder mistreatment subtypes such as psychological and physical mistreatment with suicidal ideation. We believe that future studies should be carried out to gain understanding of the association between specific subtypes of elder mistreatment and suicidal ideation.

Despite the limitations, this study has many important implications for research, policy, and community social service development. As elder mistreatment was significantly associated with an increased risk for suicidal ideation, increased research efforts should be devoted to developing effective elder mistreatment prevention and intervention programs. Community service organizations should improve education on elder mistreatment to en- 
hance public awareness of the issue. Given the mediating effect of social support on the association between elder mistreatment and suicidal ideation among older men, community organizations and family members should increase support and care for older adults who have been mistreated so as to prevent suicidal ideation arising from elder mistreatment.

From a clinical point of view, this study highlights the importance of screening for elder mistreatment among Chinese older adults. Clinicians should be knowledgeable about potential markers of elder mistreatment to facilitate its detection. When health professionals suspect elder mistreatment, detailed histories should be gathered, especially regarding psychosocial and cultural aspects. In addition to early detection, health care professionals should also improve their knowledge of reporting and treatment of elder mistreatment. Special attention should be devoted to older women who suffer from elder mistreatment, given their heightened risk for suicidal ideation.

\section{Conclusion}

This study suggests that elder mistreatment is associated with an increased risk for suicidal ideation among Chinese older adults in the USA, but that this association is only significant among older women after adjusting for all potential confounders. Longitudinal studies should be conducted to explore the mechanisms through which elder mistreatment links with suicidal ideation.

\section{Acknowledgements}

X. Dong was supported by National Institute on Aging grants (R01 AG042318, R01 MD006173, R01 CA163830, R34 MH100443, R34 MH100393, and RC4 AG039085), by a Paul B. Beeson Award in Aging, as well as by the Starr Foundation, the American Federation for Aging Research, the John A. Hartford Foundation, and the Atlantic Philanthropies.

\section{References}

1 Bonnie RJ, Wallace RB: Elder Mistreatment: Abuse, Neglect, and Exploitation in an Aging America. Washington, National Academics Press, 2003.

2 Acierno R, Hernandez MA, Amstadter AB, Resnick HS, Steve K, Muzzy W, et al: Prevalence and correlates of emotional, physical, sexual, and financial abuse and potential neglect in the United States: the National Elder Mistreatment Study. Am J Public Health 2010;100:292-297.

3 Dong X, Simon MA: Is impairment in physical function associated with increased risk of elder mistreatment? Findings from a community-dwelling Chinese population. Public Health Rep 2010;125:743-753.

4 Dong X, Simon M, Rajan K, Evans DA: Association of cognitive function and risk for elder abuse in a community-dwelling population. Dement Geriatr Cogn Disord 2011;32: 209-215.

5 Begle AM, Strachan M, Cisler JM, Amstadter $A B$, Hernandez M, Acierno R: Elder mistreatment and emotional symptoms among older adults in a largely rural population: the South Carolina Elder Mistreatment Study. J Interpers Violence 2010;26:2321-2332.

6 Mouton CP, Rodabough RJ, Rovi SL, Brzyski RG, Katerndahl DA: Psychosocial effects of physical and verbal abuse in postmenopausal women. Ann Family Med 2010;8:206-213.

7 Dong X, Simon M, Mendes de Leon C, Fulmer T, Beck T, Hebert L, et al: Elder self-neglect and abuse and mortality risk in a communitydwelling population. JAMA 2009;302:517526.
8 Corna LM, Cairney J, Streiner DL: Suicide ideation in older adults: relationship to mental health problems and service use. Gerontologist 2010;50:785-797.

9 Pfaff JJ, Almeida OP: Detecting suicidal ideation in older patients: identifying risk factors within the general practice setting. Br J Gen Pract 2005;55:269-273.

10 Yip PS, Chi I, Chiu H, Chi Wai K, Conwell Y, Caine E: A prevalence study of suicide ideation among older adults in Hong Kong SAR. Int J Geriatr Psychiatry 2003; 18:1056-1062.

11 Almeida OP, Draper B, Snowdon J, Lautenschlager NT, Pirkis J, Byrne G, et al: Factors associated with suicidal thoughts in a large community study of older adults. Br J Psychiatry 2012;201:466-472.

12 De Leo D, Krysinska K, Bertolote JM, Fleischmann A, Wasserman D: Suicidal behaviours on the five continents among the elderly; in Wasserman D, Wasserman C (eds): Oxford Textbook of Suicidology and Suicide Prevention. Oxford, Oxford University Press, 2009, pp 693-702.

13 Waern M, Beskow J, Runeson B, Skoog I: Suicidal feelings in the last year of life in elderly people who commit suicide. Lancet 1999;354: 917-918.

14 Vanderhorst RK, McLaren S: Social relationships as predictors of depression and suicidal ideation in older adults. Aging Ment Health 2005;9:517-525.

15 Alhusen JL, Frohman N, Purcell G: Intimate partner violence and suicidal ideation in pregnant women. Arch Womens Ment Health 2015;18:573-578.
16 Martin G, Bergen HA, Richardson AS, Roeger L, Allison S: Sexual abuse and suicidality: gender differences in a large community sample of adolescents. Child Abuse Negl 2004;28: 491-503.

17 Yanqiu G, Yan W, Lin A: Suicidal ideation and the prevalence of intimate partner violence against women in rural western China. Violence Against Women 2011;17:12991312.

18 Stein DJ, Chiu WT, Hwang I, Kessler RC, Sampson N, Alonso J, et al: Cross-national analysis of the associations between traumatic events and suicidal behavior: findings from the WHO World Mental Health Surveys. PLoS One 2010;5:e10574.

19 Calder J, McVean A, Yang W: History of abuse and current suicidal ideation: results from a population based survey. J Fam Violence 2010;25:205-214.

20 Chen SX, Wu WC, Bond MH: Linking family dysfunction to suicidal ideation: mediating roles of self-views and world-views. Asian J Soc Psychol 2009;12:133-144.

$21 \mathrm{Wu}$ L, Shen M, Chen H, Zhang T, Cao Z, Xiang $\mathrm{H}$, et al: The relationship between elder mistreatment and suicidal ideation in rural older adults in China. Am J Geriatr Psychiatry 2013;21:1020-1028.

22 Dong X, Li Y, Simon MA: Social engagement among US Chinese older adults - findings from the PINE Study. J Gerontol A Biol Sci Med Sci 2014;69(suppl 2):S82-S89. 
23 Dong X, Chen R, Simon MA: Anxiety among community-dwelling US Chinese older adults. J Gerontol A Biol Sci Med Sci 2014; 69(suppl 2):S61-S67.

24 Dong X, Chen R, Simon MA: The prevalence of medical conditions among US Chinese community-dwelling older adults. J Gerontol A Biol Sci Med Sci 2014;69(suppl 2):S15-S22.

25 Dong X, Chen R, Simon MA: Experience of discrimination among US Chinese older adults. J Gerontol A Biol Sci Med Sci 2014; 69(suppl 2):S76-S81.

26 Dong X, Chen R, Li C, Simon MA: Understanding depressive symptoms among community-dwelling Chinese older adults in the Greater Chicago area. J Aging Health 2014;26: 1155-1171.

27 Dong X, Chen R, Fulmer T, Simon MA: Prevalence and correlates of elder mistreatment in a community-dwelling population of US Chinese older adults. J Aging Health 2014;26: 1209-1224.

28 Dong X, Chen R, Wong E, Simon MA: Suicidal ideation in an older US Chinese population. J Aging Health 2014;26:1189-1208.

29 Yen Y-C, Yang M-J, Yang M-S, For L, Chun $\mathrm{S}$, Chen $\mathrm{H}$, et al: Suicidal ideation and associated factors among community-dwelling el- ders in Taiwan. Psychiatry Clin Neurosci 2005;59:365-371.

30 Simon MA, Chang E-S, Rajan KB, Welch MJ, Dong X: Demographic characteristics of US Chinese older adults in the Greater Chicago area assessing the representativeness of the PINE study. J Aging Health 2014;26:11001115.

31 Dong X, Wong E, Simon MA: Study design and implementation of the PINE study. J Aging Health 2014;26:1085-1099.

32 Hwalek MA, Sengstock MC: Assessing the probability of abuse of the elderly: toward development of a clinical screening instrument. J Appl Gerontol 1986;5:153-173.

33 Schofield MJ, Mishra GD: Validity of self-report screening scale for elder abuse: Women's Health Australia Study. Gerontologist 2003; 43:110-120.

34 Dong X, Beck T, Simon MA: Loneliness and mistreatment of older Chinese women: does social support matter? J Women Aging 2009; 21:293-302

35 Dong X, Simon MA, Gorbien M: Elder abuse and neglect in an urban Chinese population. J Elder Abuse Negl 2007;19:79-96.

36 Chiu HFK, Dai J, Xiang YT, Chan SSM, Leung $\mathrm{T}, \mathrm{Yu} \mathrm{X}$, et al: Suicidal thoughts and behaviors in older adults in rural China: a preliminary study. Int J Geriatr Psychiatry 2012;27:11241130.

37 American Psychiatric Association: Diagnostic and Statistical Manual of Mental Disorders. Washington, American Psychiatric Association, 1994

38 Copeland JRM, Dewey ME: Neuropsychological diagnosis (GMS-HAS-AGECAT package). Int Psychogeriatr 1991;3(suppl 1):S43S49.

39 Yeung A, Fung F, Yu SC, Vorono S, Ly M, Wu $S$, et al: Validation of the Patient Health Questionnaire-9 for depression screening among Chinese Americans. Compr Psychiatry 2008; 49:211-217.

40 Dong X, Simon MA, Gorbien M, Percak J, Golden R: Loneliness in older Chinese adults: a risk factor for elder mistreatment. J Am Geriatr Soc 2007;55:1831-1835.

41 Olofsson N, Lindqvist K, Danielsson I: Fear of crime and psychological and physical abuse associated with ill health in a Swedish population aged 65-84 years. Public Health 2012; 126:358-364.

42 Matud MP: Gender differences in stress and coping styles. Pers Individ Dif 2004;37:14011415. 